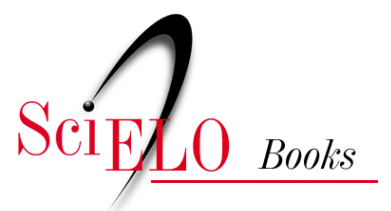

\title{
1. Gênese Sócio-Histórica da Saúde Coletiva no Brasil
}

\author{
Ligia Maria Vieira-da-Silva
}

\section{SciELO Books / SciELO Livros / SciELO Libros}

VIEIRA-DA-SILVA, L.M. Gênese Sócio-Histórica da Saúde Coletiva no Brasil. In: LIMA, N.T., SANTANA, J.P., and PAIVA, C.H.A., orgs. Saúde coletiva: a Abrasco em 35 anos de história [online]. Rio de Janeiro: editora FIOCRUZ, 2015, pp. 25-48. ISBN: 978-85-7541-590-0. Available from: doi: $10.7476 / 9788575415900.0003$. Also available in ePUB from: http://books.scielo.org/id/q4gzb/epub/lima-9788575415900.epub.

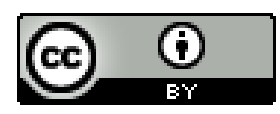

All the contents of this work, except where otherwise noted, is licensed under a Creative Commons Attribution $\underline{4.0 \text { International license. }}$

Todo o conteúdo deste trabalho, exceto quando houver ressalva, é publicado sob a licença Creative Commons Atribição 4.0. 


\title{
Gênese Sócio-Histórica dA Saúde Coletiva no BrasiL ${ }^{1}$
}

Ligia Maria Vieira-da-Silva

\begin{abstract}
A história dos saberese práticas sobre a saúdee a doença tem sido, de forma dominante, a história dos saberes e práticas da medicina e do campo médico, que tem por objeto o doente e como prática a arte, a técnica e a ciência de curar ou aliviar o sofrimento. Entrelaçados a esse campo, ora como subespaço orgânico e dominado, ora como projeto contra-hegemônico, desenvolveram-se também espaços sociais em que a produção do conhecimento e as práticas relacionadas estavam voltadas não apenas para a doença no indivíduo, mas sobretudo para o processo saúde-doença na sua dimensão coletiva. Como os problemas de saúde no âmbito populacional demandaram historicamenteumagestãoestataleumaorganizaçãoadministrativa correspondente, as ações de saúde decorrentes surgiram com o advento do Estado moderno e também contribuíram para a sua construção (Pinell, 1995).

Tais espaços sociais têm recebido diversas denominações ao longo da história: polícia médica, higiene, saúde pública, medicina social, medicina preventiva, medicina comunitária e nova saúde pública. Essas diferentes denominações são o resultado de um processo histórico de construção social e frequentemente estiveram ligadas a movimentos sociais (Rosen, 1974; Donnangelo \& Pereira, 1976; Pinell, 1992, 2002; Paim, 2006). Seu significado tem sido objeto de diversas análises: como polícia médica (Rosen, 1974); desempenhando funções de controle social, medicalização da família além de conservação da força de
\end{abstract}

\footnotetext{
Este capítulo corresponde a uma versão ampliada e modificada do artigo "The genesis of collective health in Brazil", originalmente publicado na revista Sociology of Health \& Illness (Vieira-da-Silva \& Pinell, 2014). Trabalho desenvolvido como parte das atividades de estágio sênior no Centro Europeu de Sociologia e Ciência Política (França), financiado pela Coordenação de Aperfeiçoamento de Pessoal de Nível Superior (Capes) e pelo Conselho Nacional de Desenvolvimento Científico e Tecnológico (CNPq).
} 
trabalho (Foucault, 1976); como questão de Estado (Pinell, 1995); como cultura e espaço político da saúde (Fassin\&Dozon,2001; como partede um processo demedicalizaçãonegociada (Bourdelais \& Faure, 2005).

Na América Latina verificou-se, a partir dos anos 1970, um movimento voltado para a constituição de uma medicina social que guardava identidade com a medicina social europeia do século XIX. Há controvérsias na interpretação desse fenômeno, que tem sido estudado tanto como corrente de pensamento original e específica da América Latina (Paim, 1992; Waitzkin et al., 2001; Tajer \& Laurell, 2003; Yamada, 2003; Breilh, 2008) quanto como uma das manifestações históricas de um fenômeno universal que corresponderia à busca por justiça social supostamente característica da saúde pública internacional (Krieger, 2003). Nesta segunda perspectiva, a medicina social tem sido vista como uma disciplina acadêmica presente não só na América Latina como também no mundo anglo-americano (Porter, 2006). Esses trabalhos, na sua maioria, não vêm se apoiando em investigações empíricas das experiências nacionais.

O caso brasileiro, em particular, apresenta especificidades identificadas pelos diversos estudosqueanalisaramaspectos dagênese doespaço da medicina social, aquidenominado de saúde coletiva, relacionados principalmente às características da Reforma Sanitária brasileira (Nunes, 1989; Paim, 1992, 2008; Paim \& Almeida-Filho, 1998; Paim et al., 2011) e dos movimentos sociais a ela associados (Escorel, 1999) ou à história da Associação Brasileira de Pós-Graduação em Saúde Coletiva (Abrasco) (Lima \& Santana, 2006). Em que medida essa invenção brasileira corresponde a um processo sócio-histórico específico ou apenas a uma denominação distinta de um mesmo fenômeno social? Em que medida trata-se de uma ruptura com a higiene e a saúde pública institucionalizada ou uma continuidade? Os estudos que trataram do tema têm analisado a saúde coletiva brasileira principalmente como um campo de saberes e práticas (Teixeira, 1985; Costa, 1992; Nunes, 1994; Paim \& AlmeidaFilho, 1998;Luz, 2009), porém enfatizam, em sua análise, o componente conhecimento (Garcia, 1985; Teixeira, 1985; Ribeiro, 1991; Luz, 2009). Tais estudos podem ser considerados como parte do trabalho de produção social do campo, ou seja: procuram, por vezes, analisar o que seria o paradigma da saúde coletiva, ou então assumem um conteúdo prescritivo discutindo o que a saúde coletiva deveria ser (Campos, 2000). Em alguns desses estudos, embora o conceito de campo seja remetido a Bourdieu (Ribeiro, 1991; Costa, 1992; Luz, 2009), o potencial da análise desse autor não tem sido devidamente explorado, particularmente no que diz respeito à investigação das interações entre as trajetórias individuais e as condições de possibilidade históricas.

Pretende-se suprir essa lacuna buscando investigar a gênese desse espaço social com base na teoria das práticas de Bourdieu $(1979,1996)$. A esse respeito, o referido autor, tendo identificado propriedades comuns aos diversos campos da produção cultural, considerava pertinenteentão aplicaresses princípios gerais a outros casos particulares, visandoa identificar 
as especificidades e as invariantes dos diversos universos sociais (Bourdieu, 1996). Este capítulo, ao estudar a emergência de um espaço que tem entre suas questões o projeto de se tornar campo, também pode contribuir para a análise dos processos de constituição dos habitus específicos a um determinado universo social.

\section{Aspectos MetodolóGicos}

Foi realizado um estudo sócio-histórico sobre a gênese do espaço da saúde coletiva no Brasil no período compreendido entre os anos 1960 - época caracterizada pela criação de departamentos, residências e cursos de pós-graduação em medicina preventiva e social (Nunes et al., 2010) e a emergência de um pensamento crítico sobre as relações entre saúdedoença e sociedade (Arouca, 2003; Donnangelo \& Pereira, 1976), considerados como marcos teóricos - e 1986, ano de realização da 8ª Conferência Nacional de Saúde. Realizaram-se 24 entrevistas ${ }^{2}$ entre 2008 e 2011, com duração média de duas horas, com alguns dos fundadores da saúde coletiva brasileira. Os entrevistados foram selecionados entre docentes e profissionais que integraram os principais departamentos de medicina preventiva ou que participaram diretamente da criação da então Associação Brasileira de Pós-Graduação em Saúde Coletiva (Abrasco) e ou do Centro Brasileiro de Estudos de Saúde (Cebes). Sobre os fundadores já falecidos, foram consultadas biografias publicadas e entrevistas anteriormente concedidas. Também consultaram-se os depoimentos orais depositados no Departamento de Arquivo e Documentação, Casa de Oswaldo Cruz, Fundação Oswaldo Cruz, e outros registrados pelo Projeto Memória Unirio (Unirio, 2005), o que totalizou o estudo das trajetórias de 28 fundadores (Quadro 1). A análise das trajetórias apoiou-se também na consulta aos currículos dos diversos agentes ${ }^{3}(<\mathrm{http}: / /$ lattes.cnpq.br $>$ ). Todos os entrevistados assinaram termo de consentimento informado, e o projeto foi aprovado pelo Comitê de Ética em pesquisa do Instituto de Saúde Coletiva da Universidade Federal da Bahia (ISC/Ufba).

\footnotetext{
2 Posteriormente, foram realizadas mais dezentrevistas comagentes queocupavam posições dominantes na saúde coletiva, a exemplo de editores das principais revistas científicas nacionais da área, presidentes da Abrasco, pesquisadores membros da Academia Brasileira de Ciências e dirigentes do Conselho Nacional de Secretários de Saúde (Conass).

3 O termo agente é aqui utilizado no sentido que lhe é dado por Bourdieu como agentes das práticas sociais. $O$ uso do conceito de agente é discutido por este autor como alternativa ao conceito de ator e de sujeito. Decorre de sua teoria das práticas sociais em que a ação social não é produto de um cálculo racional (Bourdieu \& Wacquant, 1992).
} 


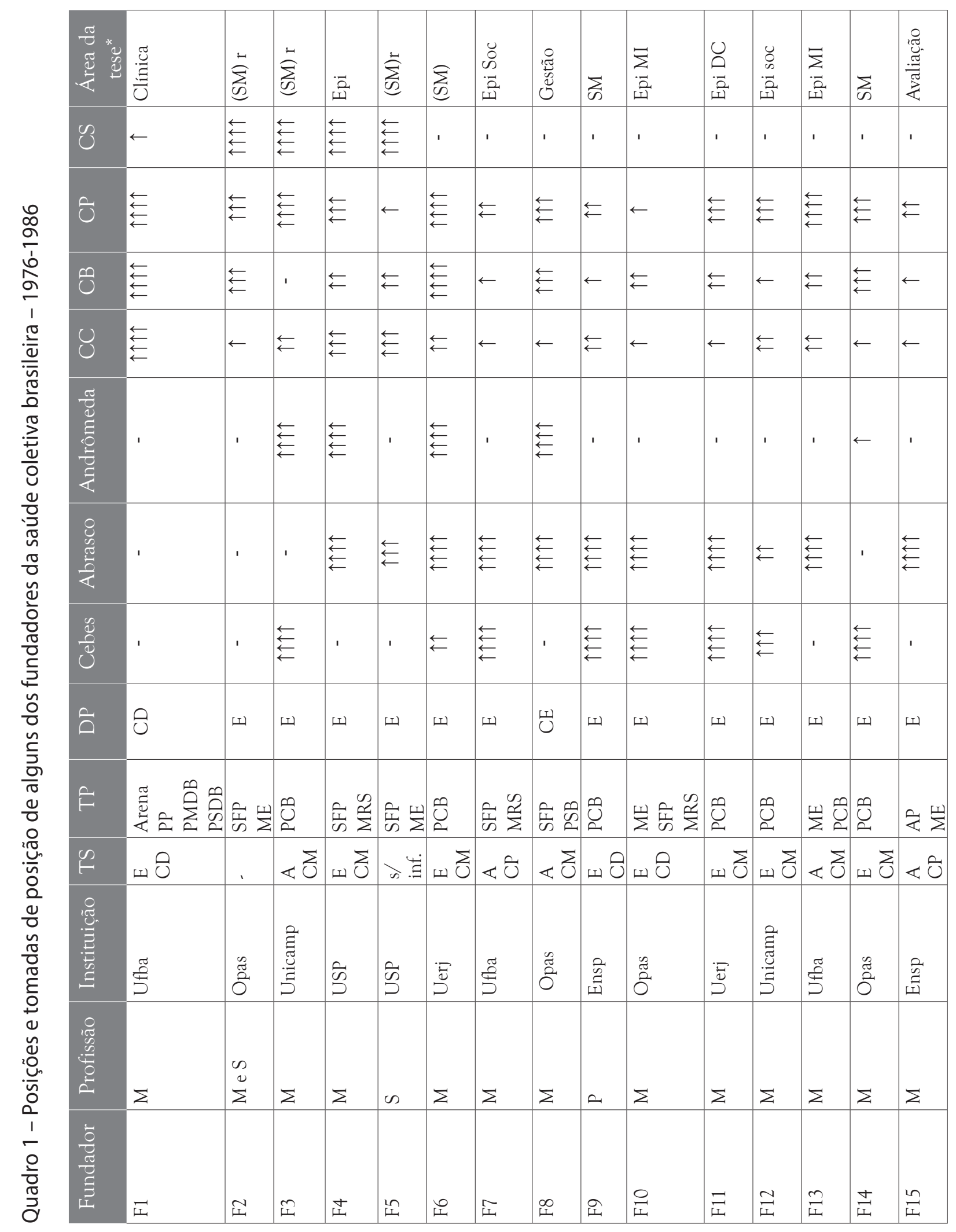




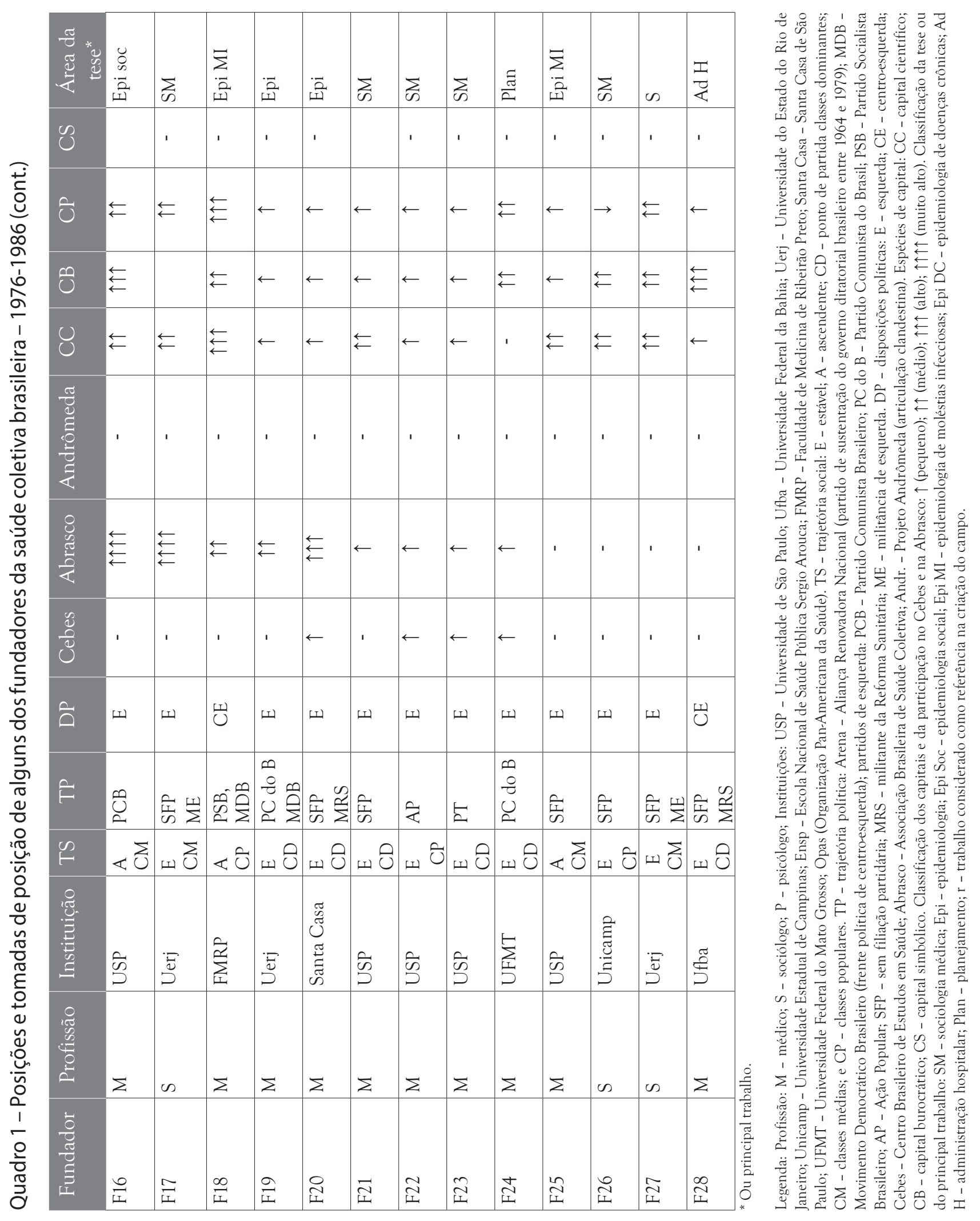


A recuperação da trajetória (social, profissional e política) de cada um dos fundadores fez-se com base em roteiros individualizados. As trajetórias sociais foram consideradas como ascendentes ou estáveis quando comparadas as profissões dos pais com a profissão dos entrevistados. Aferiu-se o ponto de partida das trajetórias por meio das profissões dos avós paternos ou maternos; a trajetória profissional, por meio das ocupações. As disposições políticas foram verificadas por meio da filiação ou militância em partidos políticos e/ou em movimentos sociais (Quadro 1).

A análise das condições de possibilidade de surgimento da saúde coletiva apoiou-se principalmente em fontes bibliográficas. Investigaram-se ainda as concepções sobre saúde coletiva, Reforma Sanitária, identidade profissional e opções acadêmicas. Particularmente pediu-se aos entrevistados que falassem sobre os eventos de constituição do campo, como a criação dos departamentos de medicina preventiva, a fundação da Abrasco e do Cebes e sobre a Reforma Sanitária. Consultaram-se também diversas fontes documentais e bibliográficas, como atas da criação da Abrasco, relatórios de encontros e biografias, artigos de autoria dos agentes entrevistados. As entrevistas foram realizadas pela autora, gravadas e transcritas e, em grande parte, revisadas pelos entrevistados; em seguida, sumarizadas em quadros individuais, contendo as trajetórias, e em quadros comparativos (Quadro 1). A análise das diversas espécies de capital foi feita por meio dos critérios relacionados no Quadro 2. O capital simbólico, capital de reconhecimento, foi aferido apenas para os fundadores falecidos mencionados pela maioria dos entrevistados como lideranças responsáveis, quer pela formulação teórica, quer pelo papel na articulação política que resultou na criação desse espaço social.

O conceito de campo de Bourdieu aqui utilizado implica a identificação das disposições comuns aos agentes que conformariam um habitus - disposições essas adquiridas ao longo de uma trajetória que corresponderia às sucessivas posições ocupadas no espaço social pelos entrevistados em certas condições históricas de possibilidade. Implica também a identificação de sua autonomia relativa em relação a outros campos e ao campo do poder (Bourdieu, 1996). Este autor usa por vezes como sinônimos os conceitos de espaço e campo (Bourdieu, 2012). Contudo, o conceito por ele sistematizado e que compõe sua teoria das práticas sociais é o conceito de campo, que está mais bem desenvolvido no livro As Regras da Arte (Bourdieu, 1996).

A caracterização de um microcosmo social como campo não é um exercício apenas teórico; requer a realização de um estudo empírico (Bourdieu \& Wacquant, 1992). Na presente investigação, o conceito de espaço social foi usado para indicar que o universo social estudado, na sua gênese, embora funcionasse como campo, no sentido de uma rede de relações entre agentes, com uma illusio ${ }^{4}$ compartilhada, não reunia todas as características

${ }^{4}$ O conceito illusio é utilizado por Bourdieu no sentido de crença, investimento, interesse ou como"ilusão de realidade coletivamente partilhada" (Bourdieu, 1996: 366), por meio do qual os agentes sociais participam e se envolvem nos jogos nos diferentes campos sociais. 


\section{de um campo consolidado, a exemplo dos campos científico e burocrático - para citar dois universos sociais investigados por Bourdieu $(1994,2012)$.}

\section{Quadro 2-Critérios para análise da composição das diferentes espécies de capital no período da gênese do espaço da saúde coletiva brasileira - 1976-1986}

\begin{tabular}{|c|c|c|c|c|}
\hline $\begin{array}{l}\text { Volume do capital } \\
\text { Tipo de capital }\end{array}$ & $\begin{array}{c}\text { Muito alto } \\
\uparrow \uparrow \uparrow \uparrow\end{array}$ & $\begin{array}{l}\text { Alto } \\
\uparrow \uparrow \uparrow\end{array}$ & $\begin{array}{l}\text { Médio } \\
\uparrow \uparrow\end{array}$ & $\begin{array}{c}\text { Pequeno } \\
\uparrow\end{array}$ \\
\hline Científico* & $\begin{array}{l}\text { Reconhecimento } \\
\text { internacional } \\
\text { Prêmios } \\
\text { internacionais }\end{array}$ & $\begin{array}{l}\text { Reconhecimento } \\
\text { nacional } \\
\text { Professor titular } \\
2 \text { ou + artigos } \\
\text { em periódicos } \\
\text { indexados/ano** }\end{array}$ & $\begin{array}{l}\text { Reconhecimento } \\
\text { local } \\
\text { Doutorado } \\
1 \text { artigo em } \\
\text { periódicos } \\
\text { indexados/ano** }\end{array}$ & Mestrado \\
\hline Burocrático & $\begin{array}{l}\text { Cargos de direção } \\
\text { em OIs (Opas, } \\
\text { OMS) e } \\
\text { no MS }\end{array}$ & $\begin{array}{l}\text { Cargos de direção } \\
\text { intermediários nos } \\
\text { diversos níveis e } \\
\text { em SES }\end{array}$ & $\begin{array}{l}\text { Cargos técnicos e } \\
\text { de assessoria em } \\
\text { OI e no MS e de } \\
\text { direção em SMS }\end{array}$ & $\begin{array}{l}\text { Cargos técnicos } \\
\text { (assessor, } \\
\text { consultor) }\end{array}$ \\
\hline $\begin{array}{l}\text { Burocrático } \\
\text { universitário }\end{array}$ & Reitor & Diretor de unidade & $\begin{array}{l}\text { Chefe de } \\
\text { departamento } \\
\text { Coordenador de } \\
\text { pós-graduação }\end{array}$ & $\begin{array}{l}\text { Coordenador de } \\
\text { disciplina }\end{array}$ \\
\hline Político & $\begin{array}{l}\text { Ministro da Saúde, } \\
\text { presidentes de OIs }\end{array}$ & $\begin{array}{l}\text { Secretários } \\
\text { estaduais de Saúde }\end{array}$ & $\begin{array}{l}\text { Secretários } \\
\text { municipais de } \\
\text { Saúde }\end{array}$ & $\begin{array}{l}\text { Outros cargos } \\
\text { técnicos de } \\
\text { indicação política }\end{array}$ \\
\hline $\begin{array}{l}\text { Participação em } \\
\text { partidos políticos }\end{array}$ & Dirigente nacional & Dirigente estadual & $\begin{array}{l}\text { Dirigente } \\
\text { municipal }\end{array}$ & Filiado \\
\hline $\begin{array}{l}\text { Participação em } \\
\text { cargos eletivos }\end{array}$ & $\begin{array}{l}\text { Senador, deputado } \\
\text { federal }\end{array}$ & Deputado estadual & Vereador & $\begin{array}{l}\text { Militante de } \\
\text { partido }\end{array}$ \\
\hline $\begin{array}{l}\text { Participação em } \\
\text { movimentos sociais }\end{array}$ & Dirigente nacional & $\begin{array}{l}\text { Dirigente estadual } \\
\text { ou participante } \\
\text { de comissões } \\
\text { nacionais de } \\
\text { associações }\end{array}$ & $\begin{array}{l}\text { Dirigente } \\
\text { municipal, ou } \\
\text { militante, de } \\
\text { comissões estaduais } \\
\text { ou municipais de } \\
\text { associações }\end{array}$ & $\begin{array}{l}\text { Filiado à } \\
\text { entidade }\end{array}$ \\
\hline
\end{tabular}

${ }^{*}$ Critérios para análise do capital científico na década de 1970.

** No período, uma publicação por ano era considerada, na área, como muito boa produtividade.

Legenda: OI - Organismo internacional; SMS - Secretaria municipal de Saúde; SES - Secretaria estadual de Saúde; MS - Ministério da Saúde. 


\section{As Condições de Possibilidade Históricas}

A construção do espaço da saúde coletiva, como todo processo de emergência de espaços sociais, écomplexo enão decorre de uma determinação direta das estruturas sociais, nem de uma ação racional planejada por um conjunto de agentes sociais que orientaram suas trajetórias nessa direção. A sua compreensão requer uma recuperação do espaço das posições e das tomadas de posição dos agentes envolvidos com a produção de saberes e práticas sobre a saúde na sociedade, bem como das condições históricas que tornaram possível a sua constituição.

Redefinições ocorridas no espaço da medicina preventiva e secundariamente da saúde pública institucionalizada foram influenciadas por alguns acontecimentos concomitantes: o financiamento das fundações americanas, a ação política e institucional da Organização Pan-Americana da Saúde (Opas), os auxílios da Financiadora de Estudos e Projetos (Finep) (Ribeiro, 1991), as contradições da conjuntura política e a situação do campo intelectual e do campo médico brasileiro nos anos 1960 e 1970. Contudo, o espaço que assim foi viabilizado resultou em local de produção de conhecimento crítico e de formulação de projetos contrahegemônicos em referência aos objetivos formalmente explicitados das próprias instituições financiadoras - aparente paradoxo, já registrado anteriormente (Escorel, 1999), e que requer interpretação.

\section{A Modernização do Ensino da Medicina e as Fundações Americanas (Kellogg, Rockefeller e Milbank)}

Aintrodução do ensino da medicina preventiva fez parte do processo de modernização da medicina iniciado com a vinda para o Brasil, em 1917, de uma missão da Fundação Rockefeller. Esta já encontrou não apenas um desenvolvimento da pesquisa em doenças infecciosas - com a escola tropicalista da Bahia (Santos, 2008) e as investigações realizadas no Rio de Janeiro e em São Paulo pelos médicos Oswaldo Cruz, Carlos Chagas, Adolfo Lutze Vital Brazil - mas também um movimento sanitarista voltado para a luta pelo saneamento, de perspectiva nacionalista e eugênica (Castro-Santos, 1989; Kobayashi et al., 2009; Hochman, 2012). Essa missão não apenas desenvolveu uma cooperação com o Departamento Nacional de Saúde Pública e os governos estaduais no combate à ancilostomíase, à malária e à febre amarela, comotambémaportou recursos consideráveisà recém-criadaFaculdadedeMedicina e Cirurgia de São Paulo, tendo posteriormente viabilizado a criação do Instituto de Higiene de São Paulo, que em 1945 viria a se transformar na Faculdade de Saúde Pública (Faria, 1999). O objetivo da Fundação Rockefeller em relação ao ensino médico era substituir o modelo francês pelo americano, com a prioridade dada ao regime de tempo integral e à pesquisa laboratorial - o que implicava a introdução de uma clínica experimental, ou seja, uma clínica apoiada na pesquisa básica. 
Na Faculdade de Medicina da Universidade Federal da Bahia - e mesmo em outras universidades do sul do país, como foi o caso da Faculdade de Medicina de Ribeirão Preto (SP) e da Universidade do Estado do Rio de Janeiro (Uerj) - esse processo ocorreu a partir da década de $1950 .{ }^{5}$ Esse movimento contou com a participação da Organização Mundial da Saúde (OMS) e foi financiado pelas fundações Kellogg, Rockefeller e Milbank, entre outras agências, que concederam bolsas de estudo a médicos recém-formados do Brasil e de outros países da América Latina para fazerem residência ou mestrado em diversas especialidades. ${ }^{6}$ A medicina preventiva estava entre as áreas priorizadas. ${ }^{7}$

\section{As Contradições da Conjuntura Política Nacional}

As principais ideias acerca do que seria a medicina social latino-americana surgiram nos anos 1960, tendo sofrido influência do clima de contestação de 1968 (Garcia, 1985). No período analisado, parte da intelectualidade latino-americana era marxista. Embora o espectro político dos agentes envolvidos com a constituição da saúde coletiva brasileira fosse amplo, incluindo partidos vinculados ao regime militar (como a Aliança Renovadora Nacional - Arena) e de oposição moderada (como o Movimento Democrático Brasileiro MDB), passando por posições políticas de centro-esquerda e disposições religiosas, diversos entrevistados eram filiados a partidos de esquerda, entre os quais o Partido Comunista Brasileiro (PCB), o Partido Comunista do Brasil (PC do B) e a Ação Popular (AP), ou tinham militância política de esquerda não organizada.

A maioria dos fundadores da saúde coletiva teve participação atuante nas lutas pela democratização do país e contribuiu para a construção de um movimento com ampla participação social, que foi a Reforma Sanitária brasileira (Paim, 2008; Escorel, 1999). Naquele período, embora o país ainda estivesse submetido a uma ditadura militar, o governo do general Geisel (1974-1979) caracterizou-se pela distensão política, além de ter implementado o 2Plano Nacional de Desenvolvimento, que incluía financiamento à pesquisa social - o que viabilizou a organização de alguns dos núcleos da emergente saúde coletiva (Ribeiro, 1991; Escorel, 1999).

\footnotetext{
5 Segundo Faria (1999), a priorização de estados do sul relacionava-se à crença da Fundação Rockefeller de que as possibilidades de desenvolvimento localizavam-se nessa área, em razão da imigração europeia. Segundo Kobayashi e colaboradores (2009), a ação da fundação entrou em sintonia com o então movimento sanitarista, que objetivava desenvolver ações de higiene e saúde pública com fins explicitamente eugênicos.

6 "A Fundação Kellogg criou, então, um programa para identificar, nos países latino-americanos, prováveis futuras lideranças na área da saúde a fim de oferecer-Ihes a oportunidade de estenderem a sua formação nos ambientes universitários dos Estados Unidos. E destacou um dos seus funcionários, o médico dr. Benjamin Horning, para visitar, anualmente, a América Latina, com o intuito de entrevistar candidatos a bolsas patrocinadas pelo American College of Physicians e pela mesma fundação. Iniciando esse programa, o dr. Horning visitou a nossa Faculdade de Medicina, cujo diretor, na época, era o meu pai" (Roberto Figueira Santos, entrevista em 7 nov. 2008).

7 "fui com uma bolsa aos Estados Unidos, onde eu fiz o mestrado na Universidade Harvard entre 1957 e 1959 (...) em um programa especial para formação de docentes em medicina preventiva que a universidade organizara atendendo a pedidos da OMS" (Rodrigues da Silva, s.d.).
} 


\section{A Composição do Espaço nas Suas Origens: os principais grupos fundadores, disposições e trajetórias iniciais}

A saúde coletiva pode serconsiderada como uma ruptura teóricae um projeto de ruptura em relação às práticas e à organização dos serviços de saúde então hegemônicas no país nos anos 1970.Três subespaçosestiveramimplicadosnesseprocesso:os departamentosdemedicina preventiva das faculdades de medicina, algumas instituições governamentais e internacionais, como o Ministério da Saúde, secretarias estaduais e municipais de Saúde e a Opas.

Guilherme Rodrigues da Silva (1928-2006), filho de agricultor e garimpeiro da região da Chapada Diamantina, na Bahia, e Hesio Cordeiro, filho de médico do Rio de Janeiro ambos médicos recém-formados que haviam orientado suas carreiras inicialmente para a clínica -, as converteram para a medicina preventiva a partir dos convites recebidos para frequentarem cursos de pós-graduação nos Estados Unidos, com financiamento da United States Agency for International Development (Usaid) - Agência dos Estados Unidos para o Desenvolvimento Internacional - e da Coordenação de Aperfeiçoamento de Pessoal de Nível Superior (Capes) nas décadas de 1950 e 1960, respectivamente, dentro de programas voltados para a modernização do ensino médico.

A terceira liderança médica foi Antonio Sergio da Silva Arouca (1941-2003), que, ao contrário dos outros dois, tinha fortes contradições com sua opção inicial pela medicina. Arouca, que pode ser considerado um dos mais importantes fundadores, teve uma trajetória singular (Marques, 2007; Franco Netto \& Abreu, 2009). De todos os fundadores, foi um dos poucos que, anos depois, saiu radicalmente do campo por ele criado para priorizar o exercício profissional da política, sucessivamente como deputado federal, candidato a vicegovernador e a vice-presidente da República, tendo sido uma das lideranças nacionais do PCB e posteriormente do Partido Popular Socialista (PPS). Dessa forma, suas disposições eram principalmente políticas. Sua opção pela medicina preventiva ocorreu quando ele vislumbrou essa escolha como uma forma de conciliar a atividade política com a medicina (Arouca, 2002).

Também para vários outros médicos, de trajetórias sociais variadas, na sua maioria estáveis, as disposições políticas foram mencionadas como razão para ingresso nos departamentos de medicina e parecem ter sido adquiridas principalmente nos meios secundaristas e universitários dos anos 1950 e 1960, já que poucos comentaram ter recebido influências familiares a esse respeito.

A opção pela pesquisa também foi uma das motivações da ida para os departamentos de medicina preventiva de alguns dos seus primeiros docentes. Cabe lembrar que essa disciplina fazia parte da renovação do ensino da medicina apoiado na pesquisa, no laboratório e no trabalho extramuros. Por essa razão, diversos entrevistados iniciaram suas carreiras em especialidades clínicas (psiquiatria, moléstias infecciosas, pediatria, entre outras), migrando depois para a medicina preventiva como forma de exercer a profissão de maneira diferente, 
comprometida com os interesses da população: uma medicina social. Outros expressavam claramente contradições com a clínica e o gosto pela gestão ou pelos números. Essas opções não corresponderam a um desenvolvimento da higiene nem da saúde pública tradicional, mas uma ruptura com estas disciplinas. Alguns dos entrevistados que cursaram disciplinas de higiene existentes no período anterior à criação dos departamentos de medicina preventiva expressavam o seu desgosto com essa disciplina.

Por sua vez, esses médicos, considerando importante introduzir o ensino das ciências sociais nas escolas médicas e também desenvolver a pesquisa sobre os determinantes sociais da saúde e da doença, estabeleceram relações com um grupo universitário de ciências sociais igualmente envolvido, em certa medida, com a militância política de esquerda, em graus variados. Para os sociólogos, o motivo aglutinador foi a constituição de uma área de conhecimento em torno das ciências sociais em saúde. Essa complementaridade de capitais (político e científico) contribuiu para delinear a futura estrutura e uma das principais oposições no interior do espaço em processo de criação.

A principal liderança desse grupo foi Maria Cecília Ferro Donnangelo (1940-1983), cujas teses de doutorado e livre-docência, Medicina e Sociedade (Donnangelo, 1975) e Saúde e Sociedade (Donnangelo \& Pereira, 1976), estão entre os primeiros trabalhos críticos que, juntamente com o ensaio de Garcia (1972), continham os elementos necessários para a ruptura teórica que iria se consolidar posteriormente com a tese de doutorado de Arouca, defendida em 1975: O Dilema Preventivista (Arouca, 2003). Nesse trabalho, o autor fazia uma crítica radical à medicina preventiva e desenvolvia a análise iniciada por Donnangelo acerca das relações entre a medicina e o capitalismo. Ambos os trabalhos inauguram um conjunto de produções acadêmicas que se seguiram na perspectiva da elaboração de uma teoria social da medicina, para uns, e da saúde, para outros. Uma teoria capaz de orientar uma ruptura também com as práticas sanitárias expressas no projeto da Reforma Sanitária.

Ambos os grupos de médicos e de sociólogos tinham, por ocasião da gênese do espaço, capitais variados. As principais lideranças eram possuidoras de elevado capital científico, burocrático ou político, diferenciando-se principalmente no que diz respeito à posição política, ao capital burocrático universitário, ao capital burocrático institucional e à geração (Quadro 1). Parte do grupo dos médicos tinha uma militância organizada principalmente no PCB, mas também em outros partidos de esquerda, como o PC do B e a Ação Popular, que era uma organização de esquerda com influência religiosa. Havia também um grupo de médicos de esquerda sem filiação partidária.

As posições políticas da geração que se graduou na década de 1950 iam de centrodireita e centro-esquerda até a esquerda católica. Já o grupo dos cientistas sociais ou não tinha militância política ou tinha militância de esquerda não partidária. Essas posições políticas guardavam uma relação com as tomadas de posição em termos acadêmicos. Assim, as posições situadas mais à esquerda no espectro político se inclinaram na direção de produzir 
teses que iriam da sociologia pura à sociologia médica e epidemiologia social. À medida que se caminhava na direção do centro do espectro político, os trabalhos de investigação incluíam a epidemiologia, a epidemiologia das doenças infecciosas, a clínica e a gestão (Quadro 1).

\section{A Construção de um Espaço Social}

Parte importante, se não central, da constituição do espaço da saúde coletiva foi a articulação entre a produção teórica crítica e a formulação de um projeto de transformação dos serviços desaúderelacionadocomaslutas pelademocratizaçãodo país. Dessaforma,omovimento por uma Reforma Sanitária que emergiu durante os anos da ditadura militar foi formulado, em grande parte, pelos pioneiros da saúde coletiva tanto em espaços de discussão e articulação clandestinos, como o PCB ou o denominado projeto Andrômeda, como nos departamentos de medicina preventiva e associações legais, como o Cebes e a Abrasco. O projeto Andrômeda era uma articulação clandestina de cerca de 15 a 20 pessoas, da qual participavam principalmente médicosqueocupavam postos emorganismosinternacionaisoueminstituições públicasfederais, além de alguns membros do PCB (Macedo, 2008; Goulart, 1996; Franco Netto \& Abreu, 2009). Omovimento sanitário organizado poressas associações envolveu lideranças estudantis, médicas e sindicais e culminou com a realização, em 1986, da $8^{a}$ Conferência Nacional de Saúde, na qual se discutiram as diretrizes e os princípios para uma Reforma Sanitária e para a organização subsequente do Sistema Único de Saúde - SUS (WHO, 2010; Paim et al., 2011).

O espaço da saúde coletiva foi assim constituído principalmente no interior do campo científico, porém com grande interseção com os campos político e burocrático. Durante os anos 1970, principalmentenosmunicípiosondehavia liberdaderelativa deatuação decorrente da progressiva ocupação de espaços de governo pelos partidos de oposição, desenvolveramse algumas experiências de organização dos serviços de saúde, em que foram atuar egressos dos programas de residência em medicina preventiva e social e dos cursos de saúde pública. Tais experiências municipais funcionaram como pilotos do que viria posteriormente a ser o SUS, bem como constituíram espaços de aglutinação, inserção profissional e debate para profissionais de formações diferenciadas, entre os quais vários agentes que contribuíram para o movimento sanitário e a construção do espaço da saúde coletiva.

O projeto Montes Claros, cidade do interior de Minas Gerais, foi considerado como um "laboratório da democratização da saúde"(Teixeira, 1995: 14), tendo contado com expressivos recursos da Usaid e da Superintendência do Desenvolvimento do Nordeste (Sudene) para a implantação, a partir de 1975, de uma rede de quarenta postos de saúde, bem como para o recrutamento etreinamento de 625 agentes de saúde.Coma coordenação técnica de Francisco de Assis Machado, o projeto Montes Claros serviu de base para concepção do Programa de Interiorização das Ações de Saúde e Saneamento (Piass), considerado a maior experiência de extensão de serviços de medicina simplificada em escala nacional (Santos, 1995). 
A experiência de Campinas (SP) coordenada por Nelson Rodrigues dos Santos, então secretário municipal de Saúde e articulador da criação do Conselho Nacional de Secretarias Municipais de Saúde (Conasems), serviu de exemplo para diversos outros municípios. Também David Capistrano coordenou, como secretário municipal de Saúde de Bauru (SP) e de Santos (SP), algumas das iniciativas inovadoras no período anterior à Constituição de 1988 (Capistrano-Filho \& Pimenta, 1988). Uma avaliação da descentralização da gestão da saúde em nove municípios - Cachoeiras de Macacu (RJ), Camaçari (BA), Maceió (AL), Arapiraca (AL), Uberlândia (MG), Cambé (PR), Penápolis (SP), Cotia (SP) e Ronda Alta (RS) -, além do bairro de Brasília Teimosa, em Recife (PE), considerados como casos exemplares de reorganização dos sistemas locais de saúde entre 1986 e 1988, foi feita em projeto financiado pela Opas (Almeida, 1989).

Haviaaindagruposinformais que seorganizarameminstituições públicas eorganismos internacionais em torno de projetos como o Programa de Preparação Estratégica de Pessoal de Saúde (Preps), que se constituiu em espaço privilegiado para a criação da Abrasco, a exemplo da equipe sob a coordenação de Carlyle Guerra Macedo, na Opas. ${ }^{8}$

A composição da primeira diretoria da Abrasco tinha um amplo espectro político necessário para viabilizar legalmente uma entidade, ainda no período autoritário. Tanto Frederico Simões Barbosa, nomeado presidente para fins de constituição da entidade, como Benedictus Philadelpho de Siqueira, presidente da primeira diretoria eleita, dispunham de reconhecimento nacional, respectivamente como pesquisador e como chefe de um grande departamento de medicina preventiva, mas necessariamente não partilhavam do projeto deconstituição de um novo campo. Eram, contudo, pessoas progressistas, deesquerda católica ou centro-esquerda, com ideais democráticos e que apoiavam as propostas do grupo dos fundadores. Essa articulação contou ainda com a participação de Carlyle Guerra Macedo, então coordenador do Programa de Recursos Humanos da Opas no Brasil, que coordenou uma das reuniões que resultaram na criação da Abrasco.

Após sua criação, as primeiras diretorias organizaram diversas reuniões, entre as quais 14 tiveram seus relatórios publicados, entre 1981 e 1983 para avaliar principalmente o ensino, mas também a pesquisa em saúde coletiva, saúde pública e medicina preventiva, envolvendo docentes das residências médicas e multiprofissionais, dos departamentos de medicina preventiva e social, dos programas de pós-graduação e dos cursos de especialização em saúde pública. Foram realizadas reuniões sobre o ensino da epidemiologia, da administração e planejamento em saúde, sobre o ensino das ciências sociais em saúde e também da saúde do trabalhador (Abrasco, 1982, 1983, 1984, 1986). Dessas áreas temáticas inicialmente

\footnotetext{
8 "a experiência da Opas foi fantástica (...) pela conjuntura do início do movimento sanitário, da nossa participação, um grupo muito (...) diferenciado. Com boas discussões das questões excelentes que se tinha na época. Então parecia um presente dos deuses eu estar ali. (...) O grupo era o Carlyle, como coordenador, o Alberto Pellegrini, o César Viera, a Isabel Santos, o Danilo Garcia, que era de São Paulo... o Paranaguá também, desde os anos 80, ele entrou junto comigo" (Roberto Passos Nogueira, entrevista em 13 nov. 2008).
} 
priorizadas, apenas a saúde do trabalhador não se consolidou como um dos eixos que iriam orientar o desenvolvimento subsequente da estrutura do espaço, embora tenha se firmado como importante área temática de pesquisa e intervenção. Nesses encontros, fica clara a coexistência entre os conteúdos e as práticas da saúde pública e da medicina preventiva preexistentes e o trabalho de construção social do novo espaço. Os textos de alguns dos fundadores (Paim, 1982; Donnangelo, 1983), bem como os relatórios finais dos encontros, explicitam as proposições de ruptura (Abrasco, 1982, 1983, 1984, 1986).

\section{O Espaço dos Pontos de Vista: entre rupturas e continuidades}

O que foi considerado como ruptura pelos agentes envolvidos com a gênese do espaço? Nas reuniões realizadas com docentes dos departamentos de medicina preventiva de São Paulo, ocorridas entre 1969 e 1973, discutiam-se o objeto da medicina preventiva, estratégias de ensino, a crítica ao planejamento normativo, o ensino das ciências sociais no curso médico (Arouca, 2003). Além disso, estavam em jogo duas concepções sobre o projeto inicial que correspondiam a dois móveis de aglutinação: o primeiro, relacionado com a crítica à medicina preventiva como proposta de mudança das práticas médicas ea alternativa de uma medicina social como possibilidade de intervenção não apenas sobre os corpos biológicos enfermos, mastambém sobrea sociedade, sua estrutura egoverno responsáveis pela produção das enfermidades. Já o segundo propósito estava ligado à criação de uma área de estudos sobre a sociologia da saúde.

A primeira perspectiva estava expressa no quadro teórico de referência do mestrado em saúde comunitária da Ufba, que resultou da discussão do I Encontro Nacional de PósGraduação em Saúde Coletiva, ocorrido em Salvador, em agosto de 1978 (Paim, 1982), um dos seminários de criação da Abrasco, a concepção do que seria a nova medicina social, então denominada saúde coletiva, continha vários pressupostos básicos, dentre os quais se destacam:

1. A saúde, como estado vital, setor de produção e campo de saber, está articulada à estrutura da sociedade por meio das suas instâncias econômicas e político-ideológicas, tendo, portanto, uma historicidade.

2. As ações de saúde (promoção, proteção, recuperação, reabilitação) constituem uma prática social e trazem consigo as influências do relacionamento dos grupos sociais.

3. O objeto da saúde coletiva é construído nos limites do biológico e do social e compreende a investigação dos determinantes da produção social das doenças e da organização dos serviços de saúde, além do estudo da historicidade do saber e das práticas sobre os mesmos. Assim, o caráter interdisciplinar desse objeto sugere uma integração no plano do conhecimento, e não no plano da estratégia de reunir profissionais com múltiplas formações. 
4. O ensino da saúde coletiva envolve a crítica permanente dos sucessivos projetos de redefinição das práticas de saúde, surgidas nos países capitalistas, que têm influenciado a reorganização do conhecimento médico e a reformulação de modelos de prestação de serviços de saúde: Reforma Sanitária, medicina social, medicina integral, medicina preventiva e medicina comunitária.

5. O processo ensino-aprendizagem não é neutro e pode ser acionado como prática de mudança ou de manutenção.

6. O conhecimento não se dá pelo contato com a realidade, mas pela compreensão das suas leis e pelo comprometimento com as forças capazes de transformá-la.

7. A participação ativa e criativa do educando e do educador no processo ensinoaprendizagempressupõeoprivilegiamentodeumapráticapedagógicafundamentalmente dialógica e antiautoritária, na qual o aluno não se limita a receber os conteúdos emitidos pelo professor.

8. O ensino de saúde coletiva remete a uma concepção ampla da prática. Nela se incluem a prática técnica, a pratica teórica e a prática política, entendidas como dimensões da prática social. Nessa perspectiva, as práticas exercidas pelos alunos e professores tendem a se articular com os movimentos mais amplos das forças sociais (Paim, 1982).

O espaço criado pelos departamentos de medicina preventiva - onde o que estava em discussão era um projeto de mudança: da medicina, do ensino médico e da prática médica ajustou-seàs disposições dealgunsdosagentesaquimencionados, quelideraramoprocesso de subversão dos propósitos dos organismos internacionais, e de alguns clínicos, desenvolvendo um pensamento crítico sobre a saúde - que iria passar a se constituir em componente de um habitus em formação e de uma illusio, ou seja, do investimento na criação do novo espaço.

O móvel de alguns sociólogos, simbolizado nas trajetórias de Donnangelo, Nunes, Madel e Loyola, entre outros, de desenvolver uma área inicialmente de sociologia da saúde, que depois converteu-se em ciências sociais em saúde, tinha grande convergência e complementaridade com o grupo de médicos. Passou, porém, a se constituir em objeto de disputas e conflitos entre disciplinas e paradigmas na base de questões da identidade do espaço. A distinção clara entreos dois propósitoseentreos dois grupos apareceexplicitamente tanto nos documentos e trabalhos sobre a gênese do campo (Donnangelo, 1975; Garcia, 1985) como também nas entrevistas. ${ }^{9}$ Para Luís Pereira (1933-1985), ${ }^{10}$ orientador da tese de doutorado de Donnangelo, ela buscava"a construção do objeto do campo de estudos que academicamente se denominaria sociologia da saúde" (Pereira, 1976: 97).

9 Na maioria das entrevistas, a resposta à pergunta "como e por que você entrou para a medicina preventiva" confirmava as duas motivações mencionadas.

${ }^{10}$ Luís Pereira fazia parte do grupo de sociólogos da Universidade de São Paulo(USP) de orientação marxista. A sociologia brasileira nos anos 1960 estava em fase de constituição. O grupo de alunos de Florestan Fernandes na USP, entre os quais destacava-se Fernando Henrique Cardoso, que viria a ser presidente da República do Brasil, construiu sua independência intelectual, no dizer de Cardoso, nos seminários sobre o capital (Garcia, 2004; Mota, Silva \& Schraiber, 2004; Bastos, 2006; Nunes, 2008). 
Essas concepções assumiram variações ou ênfases distintas, a depender da posição ocupada pelo agente. Arouca é quem explicita claramente o projeto de ruptura com a medicina preventiva e sua transformação em medicina social. Ele se torna um dos principais articuladores políticos do projeto da Reforma Sanitária brasileira: "Aí se deu essa ideia de começarmos a criar um campo no Brasil que supera a medicina preventiva e que se transforma em medicina social" (Arouca, 2002).

Já em relação a alguns agentes situados no polo institucional das organizações internacionais, como a Opas, havia um projeto para o qual a Abrasco nascera para dar vida, que tinha por objetivo mudar a organização dos serviços de saúde. Não se denominava ainda de Reforma Sanitária e era centrado na unificação e na responsabilidade estatal com a saúde (Carlyle Guerra Macedo, entrevista em 13 nov. 2008). Para um dos entrevistados, médico, com disposições mais científicas do que políticas, tratava-se de desenvolver uma epidemiologia social que superasse o estudo dos fatores de risco (Euclides Ayres Castilho, entrevista em 8 jun. 2008).

Outra diferença entre as perspectivas é que para os fundadores de trajetória iniciada na clínica e oriundos dos projetos de modernização do ensino da medicina, como Hesio Cordeiro e Guilherme Rodrigues da Silva, era a criação de uma medicina social que corresponderia ao exercício de uma clínica que incorporasse o social, mas expandisse seu objeto para os serviços de saúde. E não se tratava de criar um campo independente da medicina, com uma especificidade no que diz respeito ao objeto e à prática. Para outros, cuja trajetória revelava, desde o início, contradições com a clínica, essa era uma possibilidade não só desejável, mas possível. Nesse sentido, Hesio e Guilherme são os herdeiros que recusam a herança e contribuem para a criação das condições de subversão da ordem anterior. Fazendo residência em clínica médica em duas das mais importantes universidades do país, com lideranças clínicas, aceitam o desafio de desenvolver uma nova disciplina. Ao fazê-lo, contudo, orientados pelas disposições políticas e da rede de relações que se estabelece, contribuem definitivamente para a sua mudança. Mas também seus professores, respectivamente Piquet Carneiro e Roberto Figueira Santos, responsáveis pelas iniciativas de modernização da medicina que, diante do espaço dos possíveis-desenvolveruma carreira clínica bem-sucedida com clientela particular assegurada - optaram por trabalhar em tempo integral, articulando a clínica com a pesquisa básica.

Por fim, havia um terceiro ponto de vista dos serviços, que considerava as novas iniciativas como modernizadoras da saúde pública tradicional e viam a saúde coletiva como sinônimo da saúde pública (Uchoa \& Paim, 1982). Esses diversos enfoques se expressam na variedade de orientações conferidas aos programas de formação de pessoal naquele período. Para Garcia (1985), no fim do processo de disciplinarização da medicina social, que ocorreu em 1972-1973, existiam três tipos de programas: os programas tradicionais de saúde pública; os programas de medicina preventiva; e aqueles que buscavam a separação entre a medicina preventiva, a saúde pública e a medicina social. 


\section{O Espaço da Saúde Coletiva: entre o campo médico, o Campo do poder E O CAMPO BUROCRÁTICO}

O espaço da saúde coletiva brasileira surge, assim, como o produto do encontro de um conjunto de agentes com trajetórias diferenciadas no que diz respeito a percurso, duração e posicionamento nos campos científico, burocrático, político e do poder. Porém, com interseções e entrelaçamentos que criaram uma ordem independente de cada um deles individualmente - uma rede de relações de instituições específicas representativas, como a Abrasco; políticas, como o Cebes; e acadêmicas, como os programas de pós-graduação, os núcleos de saúde coletiva e os departamentos de medicina preventiva e social das universidades.

Ele surge no campo médico como crítica à medicina preventiva, recusa ambivalente à saúde pública institucionalizada e afirmando-se como projeto de uma medicina social cuja identidade era política com a medicina social europeia do século XIX (Nunes, 1994). A relação com o campo médico está na origem da medicina preventiva e depois em todo o desenvolvimento subsequente da saúde coletiva. Conforme analisou Arouca (2003), a medicina preventiva foi uma reação das associações médicas americanas ao crescimento da intervenção estatal no cuidado, assim como a criação dos principais departamentos de medicina preventiva ocorreu como renovação da clínica médica, e não da higiene.

Astensões entreos departamentos demedicina preventiva eas faculdades demedicina marcaram a gênese do espaço da saúde coletiva e estão presentes nos diversos depoimentos. Essas tensões se expressam na resistência dos estudantes de medicina inicialmente à higiene e depois ao ensino da medicina preventiva. Em praticamente todos os departamentos fundadores, houve experimentos com atividades práticas extramuros e com estratégias pedagógicas inovadoras visando a motivar os alunos (Teixeira et al., 1994; Paim, 1993; Vieira-da-Silva \& Paim, 1993).

O espaço aqui analisado surge como campo de conhecimento específico sobre as relações entre a saúde e a sociedade, de abordagem marxista, porém crítico, incorporando um marxismo não economicista. Busca referenciais externos alternativos como Foucault, Althusser e Bourdieu mas sem articulá-los, ou mesmo sem analisar criticamente suas contradições. A denominação saúde coletiva, embora tenha correspondido a uma estratégia de distinção possível no momento da constituição de um espaço novo que queria diferenciarse da saúde pública, da higiene e da medicina preventiva, dada a sua aparente neutralidade, passou a ser objeto de construção teórica. Como substantivo, abrigava, de um lado, alguns epidemiologistasqueapenas desejavamestudaropopulacionalabstrato; deoutro, os cientistas sociais e outros profissionais da saúde, como enfermeiros, assistentes sociais efisioterapeutas, que desejavam construir um espaço não médico. O projeto do novo espaço tinha então que enfrentarnão apenas a nova medicina preventiva, mas os habitus bem consolidados do campo médico, da saúde pública institucionalizada e do campo do poder. 
As relações dos fundadores com o campo do poder se estabelecem, no primeiro momento, a partir da formulação do projeto da Reforma Sanitária. Naquele período (1976-1986), tratava-se deum movimento contra-hegemônico,à semelhança dosmovimentos sociais que propugnavam a democratização do país. Posteriormente, com a vitória das oposições nas eleições nacionais, passou a fazer parte das questões que estavam em jogo a ocupação dos cargos de direção do SUS: de ministro a secretários estaduais e municipais cargos cuja ocupação requer íntima articulação partidária e política. Mas as relações se dão também por outra via, mais técnica, como experts do Estado, nacional e internacional (Murard \&Zylberman, 1985; Smith, 1991). Dessa forma, a hierarquização do espaço se estabeleceu, como em outros campos da produção cultural, em relação ao grau de autonomia eà distância das demandas do campo do poder - o subespaço científico, com maior autonomia, e o subespaço burocrático, mais dependente das demandas do Estado.

O aparente paradoxo, segundo o qual a crítica à medicina preventiva se desenvolve nos espaços criados para difundi-la e é financiada pelas agências que visavam a apoiá-la, na verdade revela o equívoco da priorização dos determinantes externos na explicação dos fatos sociais. A gênese do espaço da medicina social, posteriormente saúde coletiva, decorreu do encontro das trajetórias de agentes com disposições políticas variadas, porém com predominância de esquerda, que trouxeram como incorporações inconscientes a história coletiva dos intelectuais brasileiros e de alguns grupos internacionais dos anos 1960, em particular 1968: marxistas, contestadores, militância em partidos comunistas, gosto pela música popular, pela nouvelle vague e pelo Cinema Novo (Marques, 2007). Mas que incorporaram, também, a história coletiva da higiene e da saúde pública institucionalizada, da medicina e da sociologia então dominantes. Essas incorporações moduladas pelas histórias individuais resultou na variedade de trajetórias que se encontrarame construíram esse espaço novo e por isso mesmo diversificado. Por sua vez, essa heterogeneidade está na raiz de várias disputas que ocorrem no interior do espaço, principalmente no seu esforço de consolidação como campo social específico, dotado de habitus próprio e autonomia relativa ante os outros campos sociais, particularmente a medicina, as ciências sociais e as ciências básicas.

Esse aparente paradoxo relaciona-se não só com as condições de possibilidade de constituição do espaço mas também com a especificidade que vai sendo construída pelos agentesquesãoconstruídos pelasestruturasquerepresentamessas condições depossibilidade. Não bastava a criação de departamentos de medicina preventiva para se ter clínicos com olhar preventivista - isso Arouca mostrou em sua tese. Essa impossibilidade não era devida somente ao papel reprodutor da escola no sentido da análise de Bourdieu ou ao fato de o capitalismo influenciar de forma determinante as práticas médicas. Mas porque os agentes concretos que se interessaram pelo ensino da medicina preventiva, ou foram convidados a organizá-lo, trouxeram consigo as disposições para desenvolverem outras práticas teóricas e políticas que não eram aquelas. 
Também, a relação da saude coletiva com o campo do poder pode ser compreendida por meio da análise das trajetórias dos fundadores. Muitos desses percursos transitam entre um início universitário, em que há acumulação de capital científico, mas também simbólico, ea ocupação de postos em organismos internacionais diversos e cargos de primeiroe segundo escalões do governo nos diversos níveis. Essas trajetórias acompanham, em parte, a história de algumas lideranças dos movimentos sociais e democráticos que, após o fim do regime ditatorial, passaram a ocupar diversos governos estaduais, municipais e federal.

Dessa forma, a saúde coletiva é um espaço que nasce no interior do campo médico e no qual grande parte dos seus agentes guarda raízes e identidade. A perda da identidade como médico, quando existe, é ambivalente, uma vez que a nova identidade é variada: o principal expoente declara-se sanitarista - denominação dada aos anteriores experts da higienee da saúde pública institucionalizada, que o novo espaço quer romper. Apenas um dos entrevistados declarou-se socialista da saúde, denominação mais consistente com o projeto inicial. O problema da identidade não se apresentava para o grupo de cientistas sociais que objetivamente tinham um campo próprio, na época da criação da saúde coletiva. Contudo, tendo em vista que optar pela saúde coletiva correspondia a deixar seu campo de origem e ir para outro, esse grup o foi o segundo a organizar um congresso científico periódico, próprio, para delimitar seu subespaço específico.

Médico, mas não médico, sociólogo ou cientista social adjetivado: da saúde. Quadro político ambivalente (técnico para o campo político e político para o campo científico), o espaço criado tem como uma das suas questões principais afirmar-se como campo no sentido da construção da identidade e busca da autonomia relativa. Se a nova identidade não estava completamente estabelecida, as antigas também foram recusadas de forma ambivalente. Em que medida esse espaço tornou-se campo ou persiste como espaço? O estudo da sua gênese não forneceu elementos para caracterizá-lo como campo, principalmente no que diz respeito à sua autonomia relativa e à constituição de uma identidade única e um habitus específico. A resposta a essa questão requer a realização de investigações complementares, mais extensivas, com base na análise da evolução e das transformações do espaço, quase campo, da saúde coletiva no período posterior ao aqui analisado. Esta conclusão, contudo, não é contraditória com o uso corrente do conceito campo ligado à saúde coletiva, tendo em vista que faz parte da sua história a existência de um projeto de construção de um campo de saberes e práticas que tem sido continuamente retomado e redefinido. 


\section{REFERÊNCIAS}

ALMEIDA, C. M. Os Atalhos da Mudança da Saúde do Brasil: serviços em nível local - 9 estudos de caso. Rio de Janeiro: Opas, OMS, 1989.

AROUCA, A. S. S. Doutor democracia. Entrevista a Ziraldo, Zélio, Luís Pimentel, Jesus Chediak, Zezé Sack e Tatiana Molina. Pasquim, Rio de Janeiro, 28 ago. 2002. Disponível em: <http://bvsarouca.icict. fiocruz.br/imprensa.html>. Acesso em: 8 jan. 2014.

AROUCA, A. S. S. O Dilema Preventivista: contribuição para a compreensão e crítica da medicina preventiva. São Paulo, Rio de Janeiro: Unesp, Editora Fiocruz, 2003.

ASSOCIAÇÃO BRASILEIRA DE SAÚDE COLETIVA (ABRASCO). Ensino da Saúde Pública, Medicina Preventiva e Social no Brasil. v. 1. Rio de Janeiro: Núcleo de Tecnologia Educacional para a Saúde/UFRJ, Centro Latino-Americano de Tecnologia Educacional para a Saúde, Abrasco, 1982.

ASSOCIAÇÃO BRASILEIRA DE SAÚDE COLETIVA (ABRASCO). Ensino da Saúde Pública, Medicina Preventiva e Social no Brasil. v. 2. Rio de Janeiro: Núcleo de Tecnologia Educacional para a Saúde/UFRJ, Centro Latino-Americano de Tecnologia Educacional para a Saúde (Opas), Escola Nacional de Saúde Pública Sergio Arouca (Fiocruz), 1983.

ASSOCIAÇÃO BRASILEIRA DE SAÚDE COLETIVA (ABRASCO). Ensino da Saúde Pública, Medicina Preventiva e Social no Brasil. v. 3. Rio de Janeiro: Núcleo de Tecnologia Educacional para a Saúde/UFRJ, Centro Latino-Americano de Tecnologia Educacional para a Saúde (Opas), Escola Nacional de Saúde Pública Sergio Arouca (Fiocruz), 1984.

ASSOCIAÇÃO BRASILEIRA DE SAÚDE COLETIVA (ABRASCO). Estudos de Saúde Coletiva. v. 4. Rio de Janeiro: Abrasco, 1986.

BASTOS, E. R. Conversas com Sociólogos Brasileiros. São Paulo: Editora 34, 2006.

BOURDELAIS, P. \& FAURE, O. Les Nouvelles Pratiques de Santé: XVIlle-Xxe siècles. Paris: Belin, 2005.

BOURDIEU, P. La Distinction: critique social du jugement. Paris: Minuit, 1979.

BOURDIEU, P. O campo científico. In: ORTIZ, R. \& FERNANDES, F. (Orgs.). Pierre Bourdieu: sociologia. 2. ed. v. 39. São Paulo: Ática, 1994.

BOURDIEU, P. As Regras da Arte: gênese e estrutura do campo literário [1992]. Lisboa: Editorial Presença, 1996.

BOURDIEU, P. Sur l'État: cours au Collège de France (1989-1992). Paris: Seuil, 2012.

BOURDIEU, P. \& WACQUANT, L. J. D. Réponses: pour une anthropologie réflexive. Paris: Seuil, 1992.

BREILH, J. Latin American critical (social) epidemiology: new settings for an old dream. International Journal of Epidemiology, 37: 745-750, 2008.

CAMPOS, G.W. S. Saúde pública e saúde coletiva: campo e núcleo de saberes e práticas. Ciência \& Saúde Coletiva, 5(2): 219-230, 2000.

CAPISTRANO-FILHO, D. \& PIMENTA, A. L. Saúde para Todos: um desafio ao município. São Paulo: Hucitec, 1988.

CASTRO-SANTOS, L. A. D. A Fundação Rockefeller e o Estado Nacional: história e política de uma missão médico e sanitária no Brasil. Revista Brasileira de Estudos Populacionais, 6(1): 105-110, 1989.

COSTA, N. R. Ciencias sociales y salud: consideraciones sobre el nascimiento do campo de la salud colectiva en Brasil. Cuadernos Médico Sociales, 62: 36-47, 1992. 
DONNANGELO, M.C. F. Medicina e Sociedade: o médico e seu mercado de trabalho. São Paulo: Pioneira, 1975. DONNANGELO, M. C. F. \& PEREIRA, L. Saúde e Sociedade. São Paulo: Duas Cidades, 1976.

DONNANGELO, M. C. F. A pesquisa em saúde coletiva no Brasil: a década de 70. In: ASSOCIAÇÃO BRASILEIRA DE SAÚDE COLETIVA (ABRASCO). Ensino da Saúde Pública, Medicina Preventiva e Social no Brasil. Rio de Janeiro: Abrasco, Núcleo de Tecnologia Educacional para a Saúde/UFRJ, Centro Latino-Americanao de Tecnologia Educacional para a Saúde (Opas), Escola Nacional de Saúde Pública Sergio Arouca (Fiocruz), 1983.

ESCOREL, S. Reviravolta na Saúde: origem e articulação do movimento sanitário. Rio de Janeiro: Editora Fiocruz, 1999.

FARIA, L. R. O Instituto de Higiene: contribuição à história da ciência e da administração da saúde em São Paulo. Physis - Revista de Saúde Coletiva, 9(1): 175-208, 1999.

FASSIN, D. \& DOZON, J. Critique de la Santé Publique. Paris: Balland, 2001.

FOUCAULT, M. La politique de la sante au XVIIle siècle. In: FOUCAULT, M. Dits et Ecrits. Paris: Gallimard, 1976.

FRANCO NETTO, G. \& ABREU, R. Arouca, Meu Irmão: uma trajetória a favor da saúde coletiva. Rio de Janeiro: Contra Capa, Faperj, 2009.

GARCIA, A. A dependency on politics: Fernando Henrique Cardoso and sociology in Brazil. Tempo Social, 16(1): 285-300, 2004.

GARCIA, J. C. La Educación Médica en la América Latina. Washington: OPS, 1972. (Publicación científica, 255).

GARCIA, J. C. Juan Cesar Garcia entrevista Juan Cesar Garcia. In: NUNES, E. D. As Ciências Sociais em Saúde na América Latina: tendências e perspectivas. Brasília: Opas, 1985.

GOULART, F.A.A. Municipalização: veredas - caminhos do movimento municipalista de saúde no Brasil. Rio de Janeiro: Abrasco, Conasems, 1996.

HOCHMAN, G. A Era do Saneamento: as bases da política de saúde pública no Brasil [1998]. São Paulo: Hucitec, 2012.

KOBAYASHI, E. et al. Eugenia e Fundação Rockefeller no Brasil: a saúde como proposta de regeneração nacional. Sociologias, 22: 314-351, 2009.

KRIEGER, N. Latin American social medicine: the quest for social justice and public health. American Journal of Public Health, 93(12): 1.989-1.991, 2003.

LIMA, N. T. \& SANTANA, J. P. Saúde Coletiva como Compromisso: a trajetória da Abrasco. Rio de Janeiro: Editora Fiocruz, 2006.

LUZ, M. Complexidade do campo da saúde coletiva: multidisciplinaridade, interdisciplinaridade e transdisciplinaridade de saberes e práticas - análise sócio-histórica de uma trajetória paradigmática. Saúde e Sociedade, 18(2): 304-311, 2009.

MARQUES, M. Sergio Arouca: um cara sedutor. São Paulo: Brasiliense, 2007.

MOTA, A. D. S.; SILVA, J. A. \& SCHRAIBER, L. Contribuições Pragmáticas para a Organização dos Recursos Humanos em Saúde e para a História da Profissão Médica no Brasil: a obra de Maria Cecília Donnangelo. Brasília: Ministério da Saúde, 2004

MURARD, L. \& ZYLBERMAN, P. La raison de l'expert ou l'hygiène comme science sociale apliquée. Archives Européennes de Sociologie, 26(1): 58-89, 1985. 
NUNES, E. D. A medicina social no Brasil: um estudo de sua trajetória. In: CAMPOS, G. W.; MERHY, E. E. \& NUNES, E. D. Planejamento sem Normas. São Paulo: Hucitec, 1989.

NUNES, E. D. Saúde coletiva: história de uma ideia e de um conceito. Saúde e Sociedade, 3(2): 5-21, 1994.

NUNES, E. D. Cecília Donnangelo: pioneira na construção teórica de um pensamento social em saúde. Ciência \& Saúde Coletiva, 13(3): 909-916, 2008.

NUNES, E. D. et al. (2010). A pós-graduação em saúde coletiva no Brasil. Ciência \& Saúde Coletiva, 15(4): 1.923-1.934, 2010.

PAIM, J. Desenvolvimento teórico e conceitual do ensino da saúde coletiva. In: ASSOCIAÇÃO BRASILEIRA DE SAÚDE COLETIVA (ABRASCO). Ensino da Saúde Pública, Medicina Preventiva e Social no Brasil. v. 2. Rio de Janeiro: Núcleo de Tecnologia Educacional para a Saúde/UFRJ, Centro LatinoAmericano de Tecnologia Educacional para a Saúde (Opas), Ensp/Fiocruz, 1982.

PAIM, J. S. Collective health and the challenges of practice. In: PAN AMERICAN HEALTH ORGANIZATION (PAHO). The Crisis of Public Health: reflections for the debate. Washington: PAHO, 1992.

PAIM, J. S. Estratégias para integração multidisciplinar e multiprofissional na prática de saúde: 0 âmbito da graduação. Revista Baiana de Enfermagem, 2(6): 37-47, 1993.

PAIM, J. Movimentos no campo social da saúde. In: PAIM, J. S. Desafios para a Saúde Coletiva no Século XXI. Salvador: Edufba, 2006.

PAIM, J. S. Reforma Sanitária Brasileira: contribuição para a compreensão e crítica. Salvador, Rio de Janeiro: Edufba, Editora Fiocruz, 2008.

PAIM, J. S. \& ALMEIDA-FILHO, N. Collective health: a new public health or field open to new paradigms? Revista de Saúde Pública, 32(4): 299-316, 1998.

PAIM, J. S. et al. The Brazilian health system: history, advances, and challenges. The Lancet (North American edition), 377: 9-28, 2011.

PEREIRA, L. Capitalismo e saúde. In: DONNANGELO, M. \& PEREIRA, L. (Orgs.) Saúde e Sociedade. São Paulo: Livraria Duas Cidades, 1976. (Coleção História e Sociedade).

PINELL, P. Naissance d'un Fléau: histoire de la lutte contre le cancer en France (1890-1940). Paris: A. M. Métailié, 1992.

PINELL, P. Une affaire d'Etat: hygiène et le contrôle des "sauvages de l'interieur". La Recherche, 281, supl.: 20-23, 1995.

PINELL, P. La Lutte contre le Sida en France, 1981-1996. Paris: Presses Universitaires de France, 2002.

PORTER, D. How did social medicine evolve, and where is it heading? PLoS Medicine, 3(10): 1.6671.672, 2006.

RIBEIRO, P. A Instituição do Campo Científico da Saúde Coletiva no Brasil (1975-1978), 1991. Tese de Doutorado, Rio de Janeiro: Escola Nacional de Saúde Publica Sergio Arouca, Fundação Oswaldo Cruz.

RODRIGUES DA SILVA, G. Depoimento oral dado a Cristina Fonseca e Eduardo Stotz. Projeto Memória da Saúde Pública no Brasil, Departamento de Arquivo e Documentação, Serviço de Arquivo Histórico, Casa Oswaldo Cruz/Fiocruz, Rio de Janeiro, s.d. Fita cassete.

ROSEN, G. From Medical Police to Social Medicine. Essays on the History of Health Care. 1. ed. New York: New York Science History Publications, 1974.

SANTOS, A. F. D. A Escola Tropicalista da Bahia: registro de uma nova ciência na Gazeta Médica da Bahia (18661868), 2008. Dissertação de Mestrado em História da Ciências, São Paulo: Pontifícia Universidade de São Paulo. 
SANTOS, R. C. N. A história do projeto Montes Claros. In: FLEURY, S. (Org.). Projeto Montes Claros: a utopia revisitada. Rio de Janeiro: Abrasco, 1995.

$\mathrm{SMITH}$, J. A. The Idea Brokers: think tanks and the rise of the new policy elite. New York, Toronto: The Free Press, MacMillan, 1991.

TAJER, D. \& LAURELL, A. C. Latin American social medicine: roots, development during the 1990s, and current challenges. American Journal of Public Health, 93(12): 2.023-2.031, 2003.

TEIXEIRA, C. F. et al. O ensino da medicina social na graduação. Revista Brasileira de Educação Médica, 18(1): 11-19, 1994.

TEIXEIRA, S. F. As ciências sociais em saúde no Brasil. In: NUNES, E. D. (Org.) As Ciências Sociais em Saúde na América Latina: tendências e perspectivas. Brasília: Opas, 1985.

TEIXEIRA, S. F. Projeto Montes Claros: a utopia revisitada. Rio de Janeiro: Abrasco, 1995.

UCHOA, H. W. \& PAIM, E. R. A experiência da Ensp com a descentralização do curso de saúde pública. In: ASSOCIAÇÃO BRASILEIRA DE SAÚDE COLETIVA (ABRASCO). Ensino da Saúde Pública, Medicina Preventiva e Social no Brasil. Rio de Janeiro: Núcleo de Tecnologia Educacional para a Saúde, Centro Latino-Americano de Tecnologia Educacional para a Saúde, Abrasco, 1982.

UNIVERSIDADE FEDERAL DO ESTADO DO RIO DE JANEIRO (UNIRIO). Projeto Memória Unirio, 2005. Disponível em: <www.memoriasocial.pro.br/linhas/arouca/>. Acesso em: 19 abr. 2015.

VIEIRA-DA-SILVA, L. M. \& PAIM, J. S. A saúde coletiva no ensino médico: avaliação de uma disciplina em contexto restritivo. Revista Brasileira de Educação Médica, 1(17): 7-22, 1993.

VIEIRA-DA-SILVA, L. M. \& PINELL, P. The genesis of collective health in Brazil. Sociology of Health \& IIIness, 36(3): 432-446, 2014.

WAITZKIN, H. et al. Social medicine then and now: lessons from Latin America. American Journal of Public Health, 91(10): 1.592-1.601, 2001.

WORLD HEALTH ORGANIZATION (WHO). Brazil's march towards universal coverage. Bulletin World Health Organization, 88(9): 641-716, 2010.

YAMADA, S. Latin American social medicine and global social medicine. American Journal of Public Health, 93(12): 1.994-1.996, 2003.

\section{ENTREVISTAS À AUTORA ${ }^{11}$}

Anamaria Testa Tambellini. Rio de Janeiro, 27 maio 2009, 1h56min.

Carlos E. A. Coimbra Jr. Rio de Janeiro, 5 maio 2010, arquivo digital, 1h47min.

Carlyle Guerra Macedo. Brasília, 13 nov. 2008, arquivo digital, 1h55min.

Cesar Victora. São Paulo, 3 nov. 2011, arquivo digital, 1h17min.

Euclides Ayres Castilho. São Paulo, 8 jun. 2008, arquivo digital, 1h47min.

Everardo Duarte Nunes. Brasília, 24 abr. 2009, arquivo digital, 1h56min.

Fabíola de Aguiar Nunes. Brasília, 12 nov. 2008, arquivo digital, 1h56min.

11 Projeto O Espaço da Saúde Coletiva ISC/Ufba (auxílio CNPq processo n. 473126/2009-5). 
Francisco de Assis Machado. Salvador, 23 set. 2011, arquivo digital, $59 \mathrm{~min}$.

Hesio Albuquerque Cordeiro. Rio de Janeiro, 26 mar. 2009, arquivo digital, 1h16min.

Hillegonda Maria Dutilh Novaes. Novaes. São Paulo, 9 jun. 2008, aquivo digital, 2h9min.

Jairnilson Silva Paim. Salvador, 28 maio 2008, arquivo digital, 3h12min.

José Augusto Cabral de Barros. São Paulo, 15 nov. 2011, arquivo digital, 56 min.

José Carvalho Noronha. Salvador, 7 jun. 2010, arquivo digital, 1h32min.

José da Rocha Carvalheiro. Salvador, 12 maio 2011, arquivo digital, 1h30min.

José Paranaguá Santana, Brasília, 11 nov. 2008, arquivo digital, 1h49min.

Julio Muller. Salvador, 16 out. 2008, arquivo digital, 1h59min.

Lilia Blima Schraiber. São Paulo, 7 jun. 2008, arquivo digital, 1h49min.

Madel Therezinha Luz. Rio de Janeiro, maio 2009, arquivo digital, 1h 3min.

Maria Andrea Rios Loyola, Rio de Janeiro, 21 maio 2009, arquivo digital, 1h11min.

Maria Cecília de Souza Minayo. Rio de Janeiro, 28 maio 2009, arquivo digital, 1h34min.

Maria do Carmo Leal. Rio de Janeiro, 21 maio 2009, arquivo digital, 1h12min.

Moisés Goldbaum. São Paulo, 6 jun. 2008, arquivo digital, 1h39 min.

Naomar Monteiro de Almeida Filho. Salvador, 28 maio 2012, arquivo digital, 3h14min.

Paulo Eduardo Mangeon Elias. Salvador, jun. 2010, arquivo digital, 1h33min.

Paulo Marchiori Buss. Salvador, 29 set. 2009, arquivo digital, 56 min.

Reinaldo Felipe Nery Guimarães. Brasília, 18 out. 2010, arquivo digital, 1h36min.

Rita Barradas Barata. Salvador, 8 mar. 2010, arquivo digital, 1h 40min.

Roberto Figueira Santos. Brasília, 7 nov. 2008, arquivo digital, 2h5min.

Roberto Passos Nogueira. Brasília, 13 nov. 2008, arquivo digital, 1h56min.

Sebastião Antônio Loureiro de Souza e Silva. São Paulo, 2 jun. 2008, arquivo digital, 1h45m.

Sonia Maria Fleury Teixeira. Rio de Janeiro, 25 mar. 2009, arquivo digital, 1h11min. 